\title{
Acidemia propiónica en prematuro con infección asociada. Informe de caso
}

\section{Propionic acidemic in preterm baby with associated infection. A case report}

\author{
Natalia Saldaña-García, ${ }^{1}$ María del Carmen López-Castillo, ${ }^{1}$ Adrián Muñoz-Cruzado-Rosete, ${ }^{2}$ Tomás Sánchez- \\ Tamayo $^{3}$
}

\section{Resumen}

ANTECEDENTES: Las acidemias orgánicas suponen el 30\% de los errores innatos del metabolismo y pueden ser mortales en el periodo neonatal.

CASO CLínICO: Recién nacida, por cesárea urgente por placenta previa con hemorragia materna sin factores de riesgo infeccioso a las 32 semanas de gestación, de 1970 gramos. Se diagnosticó infección nosocomial y ante la falta de respuesta al antibiótico se solicitó un hemograma que reportó, como dato sobresaliente: hiperamoniemia de 1920 $\mu \mathrm{M} / \mathrm{L}$. Se dejó en ayuno y se inciaron aportes elevados intravenosos de glucosa, soporte ventilatorio invasivo, tratamiento con quelantes de amonio y depuración extrarrenal. Pese al tratamiento, falleció a las 13 horas del diagnóstico de hiperamoniemia en el cuarto día de vida. El estudio bioquímico reportó: elevación de ácido 3-hidroxipropiónico, ácido metilcítrico, propionilglicina en orina y propionil-carnitina y descenso de carnitina libre en sangre, con lo que se confirmó el diagnóstico de acidemia propiónica. En el análisis genético se detectó una mutación patogénica en homocigosis en el gen PCCB. La autopsia reveló: infección pulmonar por Enterobacter cloacae y la metabolopatía como causa de la muerte.

CONCLUSIONES: La acidemia propiónica es consecuencia de un déficit de la propionilCoA carboxilasa que interviene en la vía metabólica del propionato. Es una enfermedad autosómica recesiva, por mutación en los genes PCCA y/o PCCB. La presentación más frecuente es neonatal, con síntomas inespecíficos en la primera semana. Así, ante un neonato con síntomas inespecíficos, con respuesta inadecuada al tratamiento, a pesar de encontrar una causa que justifique el cuadro, debe sospecharse una enfermedad metabólica.

PALABRAS CLAVES: Hiperamoniemia; neonatal; ácido metilcítrico; propionil-CoA; propionil-carnitina; acidemia propiónica; PCCA; mutación; autopsia; causa de muerte.

\section{Abstract}

BACKGROUND: Organic acidemias account for $30 \%$ of inborn errors of metabolism and can be fatal in the neonatal period.

CLINICAL CASE: Newborn, born by emergency cesarean section due to placenta previa with maternal hemorrhage without infectious risk factors at 32 weeks of gestation, weighing 1970 grams. Nosocomial infection was diagnosed and due to the lack of response to antibiotics, a hemogram was requested, which reported, as outstanding data: hyperammonemia of $1920 \mathrm{M} / \mathrm{L}$. He was left fasting and was started on high intravenous glucose, invasive ventilatory support, treatment with ammonium chelators and extrarenal depuration. Despite treatment, he died 13 hours after diagnosis of hyperammonemia on the fourth day of life. The biochemical study reported: elevation of 3-hydroxypropionic acid, methylcitric acid, propionylglycine in urine and propionylcarnitine and decrease of free carnitine in blood, which confirmed the diagnosis of propionic acidemia. Genetic analysis revealed a homozygous pathogenic mutation in the PCCB gene. Autopsy revealed: Enterobacter cloacae pulmonary infection and metabolopathy as cause of death.

\footnotetext{
${ }^{1}$ Licenciada en Medicina y adjunta a la sección de Neonatología.

Médico interno residente de cuarto año. Sección de Neonatología. https:// orcid.org/0000-0002-1298-7047

${ }^{3}$ Licenciado en Medicina y jefe de la sección de Neonatología.
}

Hospital Regional Universitario de Málaga y Hospital Materno Infantil de Málaga, España.

Recibido: 22 de agosto 2020

Aceptado: 26 de marzo 2021

\section{Correspondencia}

Adrián Muñoz-Cruzado Rosete

adrianmcr1992@gmail.com

Este artículo debe citarse como: Saldaña García N, López Castillo MD, Muñoz Cruzado Rosete A, Sánchez Tamayo T. Acidemia propiónica en prematuro con infección asociada. Informe de caso. Acta Pediatr Méx 2021; 42 (3): 112-20. 
CONCLUSIONS: Propionic acidemia results from a deficit of propionyl-CoA carboxylase involved in the propionate metabolic pathway. It is an autosomal recessive disease, due to mutation in the PCCA and/or PCCB genes. The most frequent presentation is neonatal, with nonspecific symptoms in the first week. Thus, in the case of a neonate with nonspecific symptoms, with inadequate response to treatment, despite finding a cause that justifies the picture, a metabolic disease should be suspected.

KEYWORDS: Hyperammonemia; Neonatal; Methylcitric acid; Propionyl CoA; Propionyl carnitine; Propionic acidemia; PCCA; Mutation; Autopsy; Cause of death.

\section{ANTECEDENTES}

Los errores innatos del metabolismo en etapa neonatal son raros, aunque en conjunto conforman un porcentaje significativo de la patología neonatal, con una incidencia de 1 caso en cada 800 a 2500 nacimientos. ${ }^{1}$ Si bien los errores innatos del metabolismo más frecuentes son los trastornos del ciclo de la urea, las acidemias orgánicas representan el $30 \%$ y pueden ser letales en el periodo neonatal. Se caracterizan por la acumulación tóxica de ácidos orgánicos (isovalérico, propiónico y metilmalónico) e incremento de su excreción urinaria debido a un déficit enzimático en las vías de degradación de los aminoácidos. ${ }^{2-5}$

El diagnóstico temprano es decisivo para evitar secuelas neurológicas permanentes; sin embargo, la mayoría de los neonatos permanecen asintomáticos o con síntomas inespecíficos atribuibles a un proceso infeccioso u otras causas, por lo que es común la demora diagnóstica. Los síntomas aparecen luego de la acumulación de compuestos tóxicos cercanos al bloqueo metabólico, que debe sospecharse ante el deterioro inesperado e inexplicable de un paciente previamente sano con falta de respuesta al tratamiento. .,6,7,8 $^{-1}$

\section{CASO CLÍNICO}

Recién nacida, por cesárea urgente por placenta previa con hemorragia materna sin factores de riesgo infeccioso a las 32 semanas de gestación, de 1970 gramos. Los padres informaron no tener antecedentes de muertes en el periodo neonatal, abortos de repetición o ser ellos consanguíneos. La recién nacida se trasladó a la unidad de cui- dados intensivos por insuficiencia respiratoria desde el nacimiento, para soporte con presión positiva continua en la vía aérea, que se retiró a las 48 horas. Se inció la nutrición enteral por sonda nasogástrica en las primeras 6 horas, con tamizajes infecciosos a las 6 y 24 horas de vida que se reportaron negativos.

En la exploración física no se encontraron rasgos dismórficos.

Al segundo día de vida tuvo vómitos y exploración abdominal anodina, con radiografía de abdomen normal. Al tercer día experimentó hipotonía generalizada y distensión abdominal con ecografía abdominal con leve hepatomegalia, ascitis y nefromegalia leve bilateral y química sanguínea con neutropenia moderada (770 neutrófilos $/ \mathrm{m}^{3}$ ).

Se obtuvo una vía periférica y se indicó dieta absoluta, con disminución de los síntomas digestivos. Ante la persistencia de la hipotonía generalizada y escasa respuesta a los estímulos, se repitieron los exámenes de sangre, que reportaron: elevación de la proteína $\mathrm{C}$ reactiva hasta $14.5 \mathrm{mg} / \mathrm{L}$. Se inció el tratamiento empírico con antibiótico. Comenzó la respiración acidótica sin disminución de los síntomas neurológicos. Los análisis de amonio, equilibrio ácido base y cuerpos cetónicos reportaron $1920 \mu \mathrm{M} / \mathrm{L}$ de amonio, acidosis metabólica con pH 7.28; $\mathrm{PCO}_{2}$ $37 \mathrm{mmHg}$; $\mathrm{HCO}_{3} 16.5 \mu \mathrm{M} / \mathrm{L}$; exceso de bases -10.5 $\mu \mathrm{M} / \mathrm{L}$, cuerpos cetónicos positivos y anión GAP 23. El estudio se completó con ecografía craneal que mostró áreas hiperecogénicas en los núcleos de la base, tálamo y mesencéfalo sugerentes de errores innatos del metabolismo. 
Se obtuvieron muestras para estudio de errores innatos del metabolismo: ácidos orgánicos y aminoácidos en sangre y orina y determinación de acilcarnitina en sangre. Se intentó la punción lumbar, sin obtención de muestra. Se dejó en ayunas e inició el tratamiento para hiperamoniemia: quelante del amonio, benzoato sódico intravenoso con dosis de carga de $250 \mathrm{mg} / \mathrm{kg}$ y dosis de mantenimiento de $250 \mathrm{mg} / \mathrm{kg} / \mathrm{d}$ ía en perfusión continua; con dos estimulantes del ciclo de la urea: L-arginina intravenosa con dosis de carga de $350 \mathrm{mg} / \mathrm{kg}$ y $600 \mathrm{mg} / \mathrm{kg} / \mathrm{día}$ en perfusión continua, y $\mathrm{N}$-acetilcarbaglúmico oral a $200 \mathrm{mg} / \mathrm{kg}$ en dosis de carga y $100 \mathrm{mg} / \mathrm{kg}$ cada 6 horas, más carnitina intravenosa como cofactor a $50 \mathrm{mg} / \mathrm{kg}$ en dosis de carga y $75 \mathrm{mg} / \mathrm{kg} / \mathrm{día}$ cada 6 horas. Se decidió, con el fin disminuir los líquidos intravenosos, preparar conjuntamente el benzoato sódico junto con la L-arginina. Se inició la depuración extrarrenal con diálisis peritoneal y soporte con diuréticos. Se administró glucosa a $10 \mathrm{mg} / \mathrm{kg} / \mathrm{min}$ y, ante la gravedad del paciente, soporte ventilatorio invasivo.

A pesar del descenso de amonio hasta $228 \mu \mathrm{M} / \mathrm{L}$, luego de 10 horas de tratamiento, empeoró la acidosis metabólica con pH 7.03; $\mathrm{PCO}_{2} 37$ $\mathrm{mmHg} ; \mathrm{HCO}_{3}$ 10.8; exceso de bases -10.5 y ácido láctico en $2.9 \mathrm{mmol} / \mathrm{L}$ y se asoció hiperglucemia de $650 \mathrm{mg} / \mathrm{dL}$ que requirió perfusión de insulina hasta $0.8 \mathrm{UI} / \mathrm{kg} / \mathrm{h}$.

La evolución fue tórpida pese al soporte establecido, con datos de discreta sobrecarga de volumen en la ecocardiografía y dificultad para mantener adecuada la oxigenación. Sufrió un paro respiratorio resistente a las maniobras de resucitación cardiopulmonar avanzada, por lo que se produjo el desenlace mortal al cuarto día de vida a las 13 h del diagnóstico de hiperamoniemia.

La acidemia propiónica se diagnosticó con base en los reportes del estudio metabólico que informaron: importante elevación de ácido 3-hidroxipropiónico (3363 $\mu \mathrm{M} / \mathrm{L} / \mathrm{mol}$ creatinina), ácido metilcítrico (10349 $\mu \mathrm{M} / \mathrm{L}$ de creatinina) y propionilglicina ( $94 \mathrm{mM} / \mathrm{L}$ creatinina) en orina y aumento de propionil-carnitina $(13.72 \mu \mathrm{M} / \mathrm{L})$ y descenso de carnitina libre $(6.62 \mu \mathrm{M} / \mathrm{L})$ en sangre. Lo anterior se confirmó mediante el análisis de los genes en el que se detectó la mutación patogénica severa c.218_1231del14ins12 en homocigosis en el gen PCCB con padres portadores sanos. La autopsia clínica reveló, como causa de la muerte, los daños provocados por la acidemia propiónica, asociada con infección pulmonar por Enterobacter cloacae, con hemocultivo negativo.

\section{DISCUSIÓN}

La acidemia propiónica es un trastorno del metabolismo debido a un déficit enzimático de la propionil-CoA carboxilasa, enzima mitocondrial y dependiente de biotina que permite el paso de la propionil-CoA a metilmalonil-CoA. Forma parte de la vía metabólica de algunos aminoácidos ramificados: isoleucina, valina, metionina y treonina e interviene en la metabolización de los ácidos grasos de cadena impar. La incidencia de la enfermedad es de 1 caso por cada 100,000 nacidos vivos. Se debe a una mutación en el gen PCCA o PCCB, que codifican las subunidades $\alpha$ y $\beta$ de la propionil-CoA carboxilasa, con una herencia autosómica recesiva. Como consecuencia, se produce una acumulación intramitocondrial de propionil-CoA, cuyo exceso es transformado hacia otros metabolitos, sobre todo los ácidos orgánicos, como el metilcitrato y $3-\mathrm{OH}$ propionato, que se acumulan en la orina y en la sangre. El aumento de propionilCoA, aunque es más difícil de detectar en la sangre y en la orina por su volatilidad, es un metabolito indicador de probable defecto de la vía del propionato. El propionil-CoA origina la inhibición de la enzima $\mathrm{N}$-acetilglutamato sintetasa en el ciclo de la urea y del sistema de transporte mitocondrial de glicina, produciendo hiperamoniemia e hiperglicinemia. A su vez, el 
propionil-CoA se conjuga con la carnitina, dando lugar a un aumento de la propionilcarnitina y deficiencia secundaria de carnitina en plasma. ${ }^{2-5}$

La forma de presentación más común es la neonatal, como en la paciente del caso, con síntomas en la primera semana de vida. Esto se debe a que antes del nacimiento se produce la depuración intraútero de los intermediarios metabólicos tóxicos por parte de la madre, que progresivamente se acumulan después del nacimiento, luego de iniciarse la alimentación; se produce un daño orgánico en el cerebro, el hígado, el riñón y otros órganos. La clínica es inespecífica, como el rechazo del alimento y los vómitos, pero destacan los síntomas neurológicos con hipotonía troncal, hipertonía de extremidades, convulsiones, letargia progresiva e, incluso, coma, que puede acabar en insuficiencia multiorgánica. ${ }^{2,3,5}$

Existen otras dos formas: la crónica intermitente y la lentamente progresiva. La primera se caracteriza por síntomas digestivos y neurológicos ante situaciones de hipercatabolismo y la segunda por síntomas neurológicos que, sobre todo, avanzan de forma insidiosa. ${ }^{3,5}$

Ante la sospecha de cualquier error innato del metabolismo lo primero que debe hacerse es solicitar: gasometría, lactato, glucemia, cuerpos cetónicos, amonio, iones, transaminasas y coagulación. Enseguida, para el diagnóstico específico: estudios de sangre (aminoácidos, acilcarnitinas y actividad de biotinidasa), orina (aminoácidos, ácidos orgánicos y ácido orótico) y genética. ${ }^{6-8}$

En el Hospital Materno Infantil de Málaga, entre los años 2007 a 2019 se han atendido otros 3 casos de hiperamoniemia neonatal, con valores entre 287 y $318 \mu \mathrm{M} / \mathrm{L}$ debidos a: hiperinsulinismo congénito, encefalopatía hipóxico-isquémica y una encefalopatía epiléptica temprana, por alteración del gen KCNQ2. Cuadro 1

La paciente del caso tuvo hiperamoniemia y acidosis con cetosis y cetonuria, compatible con acidemia orgánica (Figura 1). A su vez, la neutropenia orientaba a acidemia orgánica porque es común la supresión por los intermediarios metabólicos tóxicos de la médula ósea. Con respecto al estudio de segundo nivel, tuvo elevación de la propionilcarnitina en sangre y aumento del ácido hidroxipropiónico, metilcrítico y propionilglicina en orina; por eso se diagnosticó acidemia propiónica. ${ }^{2,9}$

El tratamiento de los errores innatos del metabolismo con hiperamoniemia (más de $150 \mu \mathrm{M} / \mathrm{L}$ ), como es el caso de la acidemia propiónica, es una urgencia médica (Figura 2). Lo primero que debe hacerse es interrumpir el aporte proteico durante 24 a 48 horas y mantener un alto aporte calórico, casi siempre a expensas de hidratos de carbono, incluso asociando insulina si fuera

Cuadro 1. Casos de hiperamonemia neonatal en nuestro centro entre 2007-2019

\begin{tabular}{|c|c|c|c|}
\hline $\begin{array}{l}\text { Edad gestacional } \\
\text { y peso }\end{array}$ & Clínica & $\begin{array}{c}\text { Concentraciones } \\
\text { de amonio } \\
\text { al diagnóstico }\end{array}$ & Diagnóstico \\
\hline $\begin{array}{l}39+4 \text { semanas } \\
3720 \mathrm{~g}\end{array}$ & Crisis convulsiva & 287 micromoles/L & Hiperinsulinismo-hiperamoniemia \\
\hline $\begin{array}{l}39 \text { semanas } \\
3190 \mathrm{~g}\end{array}$ & $\begin{array}{c}\text { Encefalopatía } \\
\text { hipóxico-isquémica leve }\end{array}$ & $\begin{array}{l}144,9 \\
\text { micromoles/L }\end{array}$ & $\begin{array}{l}\text { Encefalopatia hipóxico-isquémica leve } \\
\text { por desprendimiento de placenta }\end{array}$ \\
\hline $\begin{array}{l}37 \text { semanas } \\
3550 \mathrm{~g}\end{array}$ & Crisis convulsiva & 300 micromoles/L & $\begin{array}{l}\text { Tratamiento con ácido valproico en encefalopatía } \\
\text { epiletica prezcoz con alteración del gen KCNQ2 }\end{array}$ \\
\hline $\begin{array}{l}32 \text { semanas } \\
1970 \mathrm{~g}\end{array}$ & $\begin{array}{l}\text { Hipotonía y mala } \\
\text { tolerancia digestiva }\end{array}$ & $\begin{array}{c}1920 \\
\text { micromoles/L }\end{array}$ & Acidemia propiónica \\
\hline
\end{tabular}




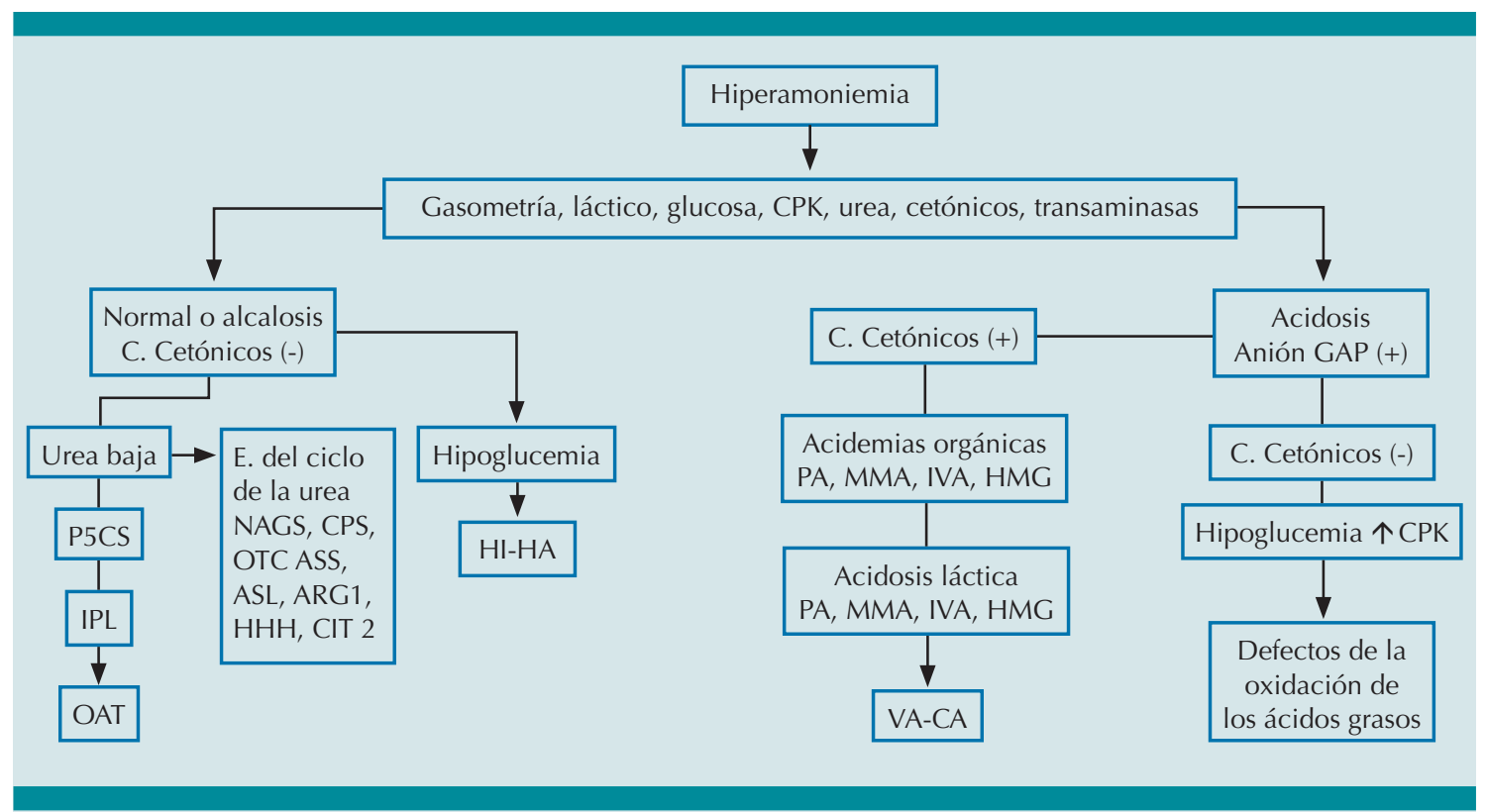

Figura 1. Diagnóstico diferencial de errores innatos del metabolismo que cursan con hiperamonemia. PA: acidemia propiónica, MMA: acidemia metilmalónica, IVA: acidemia isovalérica, HMG: aciduria 3-OH- metil glutárica, VA-CA: anhidrasa carbónica, CPK: creatin fosfokinasa, HI-HA: hiperinsulinismo-hiperamonemia, P5CS: pirrolidina 5-carboxilato sintetasa, IPL: intolerancia a proteínas con lisinuria, OAT: ornitin aminotransferasa, NAGS: $\mathrm{N}$-acetilglutamato sintetasa, CPS: carbamilfosfato sintetasa, OTC: ornitin transcarbamilasa, ASS: arginosuccínico sintetasa, ASL: arginosuccínico liasa, ARG: arginasa, HHH: hiperamonemia, hiperonitinemia, homocitrulinuria, CIT2: citrina. Extraído de: González Brabín A, Martín Rivada, Cabrero Hernández M. Neonato con rechazo de las tomas e hipoactividad. Pediatr Integral 2019; XXIII (3):162-68.

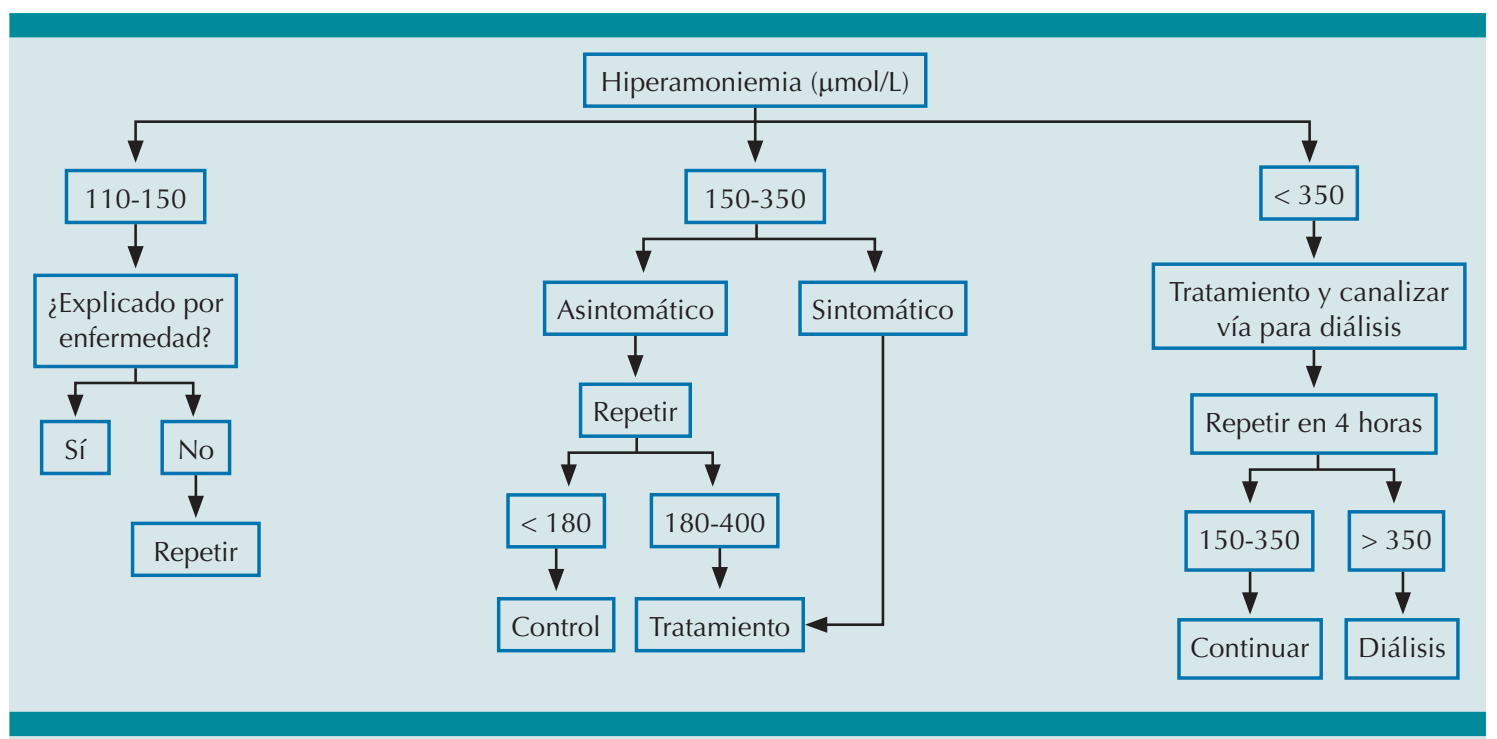

Figura 2. Tratamiento hiperamoniemia. Extraido de: Couce ML, Bustos G, García-Alix A, Lázaro A, MartínezPardo M, Molina A, et al. Guía clínica de diagnóstico y tratamiento urgente de hiperamonemia neonatal. An Pediatr. 2009;70(2):183-8. 
necesario. Si no se sospecha un defecto de la betaoxidación, que casi siempre cursa con acidosis metabólica con anión GAP aumentado, hipoglucemia y cuerpos cetónicos negativos, podrán agregarse lípidos intravenosos como fuente de energía para favorecer el anabolismo. Así, en recién nacidos, se aconseja aportar mínimo $80 \mathrm{Kcal} / \mathrm{kg} /$ día a expensas de hidratos de carbono y, si no se sospecha un defecto de la betaoxidación, a expensas de lípidos e hidratos de carbono..$^{2,6,7}$

Se dispone de diferentes fármacos que actúan en distintos sitios: estimulan el ciclo de la urea (L-arginina y ácido carbaglúmico o $\mathrm{N}$-carbamilglutamato), quelando el amonio (benzoato sódico, fenilbutirato y fenilacetato) o mejorando las vías metabólicas de otros errores innatos del metabolismo que, secundariamente, inhiben el ciclo de la urea (carnitina, hidroxicobalamina, biotina...). En todas las hiperamoniemias, sobre todo en los defectos del ciclo de la urea, debe indicarse, al menos, un quelante del amonio (benzoato sódico o fenilbutirato). A su vez, en todas las hiperamoniemias puede agregarse L-arginina para estimular el ciclo de la urea y carnitina como cofactor. En el caso de las acidemias orgánicas, defectos de la beta-oxidación, la enfermedad de la orina con olor a jarabe de arce y otras (por ejemplo, insuficiencia hepática aguda), puede agregarse $\mathrm{N}$-acetilcarbamilglutamato. ${ }^{6,7}$

Así, debe iniciarse, al menos, un quelante del amonio, como el benzoato sódico a dosis de 250 $\mathrm{mg} / \mathrm{kg}$ con una dosis de mantenimiento de 250 a $500 \mathrm{mg} / \mathrm{kg} /$ día cada 4 a 6 horas o perfusión continua. La forma intravenosa debe diluirse en $1 \mathrm{~g}$ en $50 \mathrm{~mL}$ de suero glucosado al 5 o 10\% y administrar, en un tiempo mínimo de 90 minutos, la dosis de carga. La forma oral debe administrarse con una dilución 1:10 ( $1 \mathrm{~g}$ de polvo en $10 \mathrm{~mL}$ de agua). Uno de los efectos secundarios del benzoato sódico, debido al alto contenido de sodio, es la hipernatremia, por lo que será importante efectuar controles seriados de sodio y asegurar la hidratación del paciente. ${ }^{6,7,10}$

También puede estimularse el ciclo de la urea si se administra L-arginina intravenosa a razón de 350 $\mathrm{mg} / \mathrm{kg}$ y dosis de mantenimiento de $600 \mathrm{mg} / \mathrm{kg} /$ día en perfusión continua. Debe diluirse 1 gramo en $50 \mathrm{~mL}$ de suero glucosado al 5 o 10\%; puede hacerse una dilución conjunta con el benzoato sódico. Es aconsejable administrar, mediante vía central y en un tiempo mínimo de 90 minutos, la dosis de carga. No es habitual encontrar una preparación intravenosa, por lo que la alternativa es administrar L-arginina oral a $500 \mathrm{mg} / \mathrm{kg} /$ día y, si se tiene el diagnóstico de un defecto enzimático intramitocondrial del ciclo de la urea, es suficiente con 150 a 250 mg/kg/día. ${ }^{6,7,10}$

Por último, es posible administrar carnitina por vía oral o intravenosa. En la primera se administran 50 a 300 mg/kg/día cada 6 a 8 horas y en la segunda $50 \mathrm{mg} / \mathrm{kg}$ en dosis de carga, seguido de una dosis de mantenimiento de 50 a $300 \mathrm{mg} / \mathrm{kg} /$ día en infusión continua o cada 4 a 6 horas. ${ }^{6,7,10}$

Luego de dos horas de tratamiento se cuantifica la anonimia y, si aumenta o, inicialmente, era mayor de $350 \mu \mathrm{M} / \mathrm{L}$, debe indicarse ácido carglúmico o N-carbamil glutamato por vía oral, especialmente útil en las acidemias orgánicas. Se administrarán 100 a $250 \mathrm{mg} / \mathrm{kg}$ en dosis de carga y 100 a 200 mg/kg/día cada 6 a 8 horas. $^{7}$

Debe tenerse en cuenta que existen fármacos y presentaciones de difícil acceso, como es el caso del benzoato sódico intravenoso, el fenilbutirato y fenilacetato intravenoso y oral o la L-arginina por vía intravenosa en México.

Las medidas de depuración extrarrenal están indicadas en tres casos: ${ }^{2,7}$

1. Amonio: más de $350 \mu \mathrm{M} / \mathrm{L}$ sin mejoría con medidas nutricionales y farmacológicas en 4 horas. 
2. Amonio: más de $250 \mu \mathrm{M} / \mathrm{L}$ y encefalopatía sin mejoría con medidas nutricionales y farmacológicas en 4 horas.

3. Amonio: más de $500 \mu \mathrm{M} / \mathrm{L}$.

Las principales opciones son: ${ }^{6,7}$

- Hemodiafiltración venovenosa continua: es la técnica de elección. En neonatos se utilizan catéteres 6.5 a $7 \mathrm{Fr}$ en la vena yugular interna o catéter $5.5 \mathrm{Fr}$ en la vena femoral. Por lo general, se invierten los flujos de las luces en los neonatos y se asume cierta recirculación para evitar que la succión por una luz proximal se ocluya con la pared del vaso. Permite un aclaramiento de $20 \mathrm{~mL} / \mathrm{min} / \mathrm{m}^{2}$.

- Diálisis peritoneal: es el tratamiento más indicado en niños con menos de $3 \mathrm{~kg}$. Puede aplicarse como vía puente hasta la obtención de vías para hemodiafiltración venovenosa continua. Permite un aclaramiento de 3 a $5 \mathrm{~mL} / \mathrm{min} / \mathrm{m}^{2}$.

La hemodiálisis intermitente es la técnica más eficaz pero peor tolerada en pacientes con menos de $5 \mathrm{~kg}$, por el riesgo de hipotensión. ${ }^{6,7,11} \mathrm{Si}$ se inician las medidas de depuración extrarrenal es necesario aumentar las dosis de arginina, $\mathrm{N}$-acetilcarbamilglutamato, fenilbutirato y benzoato sódico hasta en un $25 \%$.

Hoy en día se sugiere que el N-acetilcarbamilglutamato en pacientes con hiperamonemia neonatal sea de primera línea debido a que puede evitar la necesidad de depuración extrarrenal y mejorar el pronóstico a largo plazo. Esto sin dejar de tener en cuenta que el $\mathrm{N}$ acetilcarbamilglutamato tarda entre 4 a 8 horas en descender las concentraciones de amonio, tolerando concentraciones elevadas de éste hasta la acción farmacológica y evitando el inicio de medidas de depuración extrarrenal. ${ }^{6}$
El sistema de oxigenación por membrana extracorpórea está indicado en casos aislados para estabilizar al paciente y conectar al circuito de ésta la hemodiálisis. Los autores de los reportes de esos casos concluyen que en determinados pacientes con concentraciones de amonio muy elevadas (superiores a $1000 \mu \mathrm{M} / \mathrm{L}$ ), sobre todo si son recién nacidos con bajo peso y con inestabilidad hemodinámica, puede plantearse la opción del sistema de oxigenación por membrana extracorpórea para estabilizarlo y conectarlo a la hemodiálisis y conseguir flujos de diálisis mayores y un descenso del amonio más rápido, sobre todo si se trata de un paciente en situación de insuficiencia multiorgánica., ${ }^{72,13}$

En el caso de la acidemia propiónica, luego de superar la descompensación metabólica, el tratamiento crónico consiste en dieta hipercalórica y baja en aminoácidos que producen ácidos propiónicos (isoleucina, valina, metionina y treonina), manteniendo el aporte suficiente del resto de aminoácidos para no afectar la adecuada síntesis proteica y los aportes orales de carnitina para eliminar el exceso de ácido propiónico. A su vez, al ser la biotina un cofactor de la propionilCoA carboxilasa puede aportar beneficios en estos pacientes. Por último, el trasplante de hígado, o hepático-renal, está indicado en pacientes metabólicamente inestables para aportar mayor actividad enzimática, aunque hoy en día es controvertido quién y cuándo debe recibir un trasplante debido a que no es un tratamiento curativo. Todo paciente con diagnóstico de acidemia propiónica debe tener un seguimiento estrecho por parte de personal experimentado en enfermedades metabólicas con el fin de asegurar un adecuado desarrollo ponderoestatural y evitar descompensaciones metabólicas. ${ }^{2,3,14}$

En todo paciente en quien se sospeche un error innato del metabolismo deberán indagarse los antecedentes de muerte durante el periodo neonatal, de abortos de repetición y familiares 
consanguíneos. Puesto que la mayoría de los errores innatos del metabolismo son de herencia autosómica recesiva, la incidencia será mayor en comunidades altamente endogámicas, como sucede en ciertas regiones de México. A pesar de todo, aunque no tengan antecedentes, en zonas no endogámicas deberá tenerse en cuenta que un porcentaje importante son casos esporádicos por la escasa descendencia..$^{8,15}$ De manera independiente deberá practicarse el estudio genético para poder establecer el diagnóstico prenatal y preimplantacional. ${ }^{16}$ En el caso aquí informado, los padres eran portadores sanos y el análisis de los genes en el pneonato reveló la mutación c.218_1231del14ins12 en homocigosis en el gen PCCB. Esta mutación, junto con c.502G>A, se encuentra incluso en el $60 \%$ de los pacientes estudiados con acidemia propiónica y es más frecuente en población caucásica y latinoamericana. Provoca un codón de paro prematuro en la traducción del ARNm, esperándose una proteína ausente o alterada, que si se encuentra en homocigosis o en heterocigosis compuesta (es decir, un alelo con algo de actividad enzimática residual y un segundo alelo nulo) serán las mutaciones relacionadas con los fenotipos más severos. ${ }^{17,18}$

\section{CONCLUSIONES}

Los errores innatos del metabolismo tienen una clínica inespecífica y diversa; para evitar las secuelas neurológicas es decisivo el diagnóstico temprano. Así, ante un neonato con síntomas inespecíficos, a pesar de encontrar una posible causa infecciosa o de otra índole que justifique el cuadro, si existe respuesta parcial o inadecuada al tratamiento deberá sospecharse una enfermedad metabólica.

De entre los errores innatos del metabolismo, además de los trastornos del ciclo de la urea, deben tenerse en cuenta las acidemias orgánicas, como la acidemia propiónica, que es la presentación neonatal más frecuente. En los estudios de laboratorio debe sospecharse ante acidosis metabólica con cetosis o cetonuria e hiperamoniemia. Deben analizarse la sangre (aminoácidos, acilcarnitinas y actividad de biotinidasa) y la orina (aminoácidos, ácidos orgánicos y ácido orótico) e iniciar el tratamiento para hiperamoniemia.

\section{REFERENCIAS}

1. Sanderson S, Green A, Preece MA, Burton H. The incidence of inherited metabolic disorders in the West Midlands, UK. Arch Dis Child 2006; 91 (11): 896-99. http://dx.doi. org/10.1136/adc.2005.091637.

2. El-Hattab AW. Inborn Errors of Metabolism. Clin Perinatol 2015; 42 (2): 413-39. https://doi.org/10.1016/j. clp.2015.02.010.

3. Fraser JL, Venditti CP. Methylmalonic and propionic acidemias: Clinical management update. Curr Opin Pediatr 2016; 28 (6): 682-93. doi: 10.1097/MOP.0000000000000422.

4. Cammarata-scalisi F, Yen-hui C, Tze-tze L, Araque D, Callea $M$, Avenda A. Hallazgos clínicos, bioquímicos y moleculares de la acidemia propiónica. Reporte de un caso. Arch Argent Pediatr. 2019; 117 (3): 288-91. doi:10.5546/aap.2019.e288.

5. Baumgartner MR, Hörster F, Dionisi-Vici C, Haliloglu G, Karall D, Chapman KA, et al. Proposed guidelines for the diagnosis and management of methylmalonic and propionic acidemia. Orphanet J Rare Dis 2014; 9 (1): 1-36. https:// doi.org/10.1186/s13023-014-0130-8.

6. Auron A, Brophy PD. Hyperammonemia in review: Pathophysiology, diagnosis, and treatment. Pediatr Nephrol 2012; 27 (2): 207-22. https://doi.org/10.1007/s00467011-1838-5.

7. Couce ML, Bustos G, García-Alix A, Lázaro A, MartínezPardo $M$, Molina $A$, et al. Guía clínica de diagnóstico y tratamiento urgente de hiperamonemia neonatal. An Pediatr 2009; 70 (2): 183-8. https://doi.org/10.1016/j. anpedi.2008.10.007.

8. Pons MR, Vega CS. Enfoque práctico para el diagnóstico de los errores congénitos del metabolismo. Acta Pediátrica Española 1998; 56 (1): 39-56.

9. Haijes HA, van Hasselt PM, Jans JJM, Verhoeven-Duif NM. Pathophysiology of propionic and methylmalonic acidemias. Part 2: Treatment strategies. J Inherit Metab Dis 2019; 42 (5): 745-61. https://doi.org/10.1002/jimd.12128.

10. Arnold-almaraz $\mathrm{K}$, Olivares-sandoval $Z$, Revilla-estivill $N F$, Ibarra-gonz QFBI, Belmont-mart L, Vela-amieva M. Tratamiento de la hiperamonemia en pediatría. Acta Pediatr Méx 2005; 26 (6): 313-24.

11. Kim HJ, Park SJ, Park I, Lee JS, Eun HS, Kim JH, Shin JI. Korean. Acute treatment of hyperammonemia by continuous 
renal replacement therapy in a newborn patient with ornithine transcarbamylase deficiency. J Pediatr 2011; 54 (10): 425-428. doi:10.3345/kjp.2011.54.10.425.

12. Wen JX, Feldenberg LR, Abraham E, Sadiq F, Christensen KM, Braddock SR. Continuous venovenous hemodialysis via extracorporeal membrane oxygenation pump for treatment of hyperammonemia secondary to propionic acidemia in monochorionic diamniotic twin boys. J Pediatr 2016; 175: 231-2. https://doi.org/10.1016/j. jpeds.2016.05.019.

13. Summar M, Pietsch J, Deshpande J, Schulman G. Effective hemodialysis and hemofiltration driven by an extracorporeal membrane oxygenation pump in infants with hyperammonemia. J Pediatr 1996; 128 (3): 379-82.

14. Haijes HA, Jans JJM, Tas SY, Verhoeven-Duif NM, van Hasselt PM. Pathophysiology of propionic and methylmalonic acidemias. Part 1: Complications. J Inherit Metab Dis 2019; 42 (5): 730-44. https://doi.org/10.1002/jimd.12129.
15. Leonard JV, Morris AAM. Diagnosis and early management of inborn errors of metabolism presenting around the time of birth. Acta Paediatr Int J Paediatr 2006; 95 (1): 6-14. https://doi.org/10.1111/j.1651-2227.2006.tb02172.x.

16. Pérez-Cerdá $C$, Pérez $B$, Merinero $B$, Desviat $L R$, Rodríguez Pombo $P$, Ugarte $M$. Prenatal diagnosis of propionic acidemia. Prenat Diagn 2004; 24 (12): 962-4. https://doi. org/10.1002/pd.1057.

17. Pérez B, Angaroni C, Sánchez-Alcudia R, Merinero B, Pérez-Cerdá C, Specola N, et al. The molecular landscape of propionic acidemia and methylmalonic aciduria in Latin America. J Inherit Metab Dis 2010; 33 (Suppl. 2): 307-14. https://doi.org/10.1007/s10545-010-9116-4

18. Desviat LR, Pérez $B$, Pérez-Cerdá $C$, Rodríguez-Pombo $P$, Clavero S, Ugarte M. Propionic acidemia: Mutation update and functional and structural effects of the variant alleles. Mol Genet Metab 2004; 83 (1-2): 28-37. https://doi. org/10.1016/j.ymgme.2004.08.001. 